\title{
Masses and lifetimes for $r$-process nucleosynthesis: FRIB outlook
}

\author{
Rebecca Surman ${ }^{1, *}$ and Matthew Mumpower ${ }^{2, * *}$ \\ ${ }^{1}$ Department of Physics, University of Notre Dame, Notre Dame, IN USA \\ ${ }^{2}$ Theoretical Division, Los Alamos National Laboratory, Los Alamos, NM USA
}

\begin{abstract}
Nuclear masses and lifetimes are key inputs for calculations of rapid neutron capture ( $r$-process) nucleosynthesis. Masses and half-lives for thousands of nuclei from the valley of stability to the neutron drip line are required and only a fraction have been experimentally measured. Here we examine the promise of the Facility for Rare Isotope Beams, now under construction at Michigan State University, to dramatically reduce uncertainties in $r$-process abundance patterns due to uncertain masses and half-lives.
\end{abstract}

GW170817 [1] has ushered in a new era of $r$-process studies. Observations of the GW170817 neutron star merger event in the optical and infrared are interpreted to be a kilonova: an electromagnetic transient powered by the radioactive decay of heavy nuclei freshly produced via rapid neutron capture ( $r$-process) nucleosynthesis [2, 3]. This is the first clear evidence of $r$-process production and may even suggest that most of the galactic $r$-process material originates in merger events [4]. Still many questions remain. Are mergers the only site of $r$-process nucleosynthesis, or do multiple sites contribute? Can we understand neutron star merger nucleosynthesis from first principles? At what level of detail can we interpret this and future kilonova observations?

Nuclear physics plays a key role in all of the above questions. The nuclear network calculations used to estimate the nuclei produced in a merger and their associated radioactive heating rely on nuclear physics information-masses, reaction rates, $\beta$-decay and fission properties-for thousands of nuclei from the valley of stability to the neutron drip line [5, 6]. Only a fraction of the needed data has be measured experimentally, leading to large uncertainties in predicted abundance patterns [4, 6-9] and radioactive heating estimates [10].

The next generation of radioactive beam facilities will have unprecedented reach into exotic neutron-rich regions of the nuclear chart. In the United States, the Facility for Rare Isotope Beams (FRIB) is currently under construction at Michigan State University and first beam is scheduled for 2020. Here we look forward to the capabilities of FRIB and examine its potential for reducing uncertainties in simulated $r$-process abundance patterns.

Nuclear masses are of fundamental importance for $r$-process simulations. Neutron separation energies $S_{n}$ appear explicitly in the evaluation of photodissociation rates via detailed balance. $S_{n}$ set the location of the $r$-process path and shape the decay to stability, for as long as the temperature is high enough for photodissociation to compete with neutron capture and $\beta$ decay. Masses also set the $Q$-values for neutron capture and $\beta$ decay, thus influencing calculations of these quantities where experimental data is not available.

\footnotetext{
*e-mail: rsurman@nd.edu

**e-mail: matthew@mumpower.net
} 


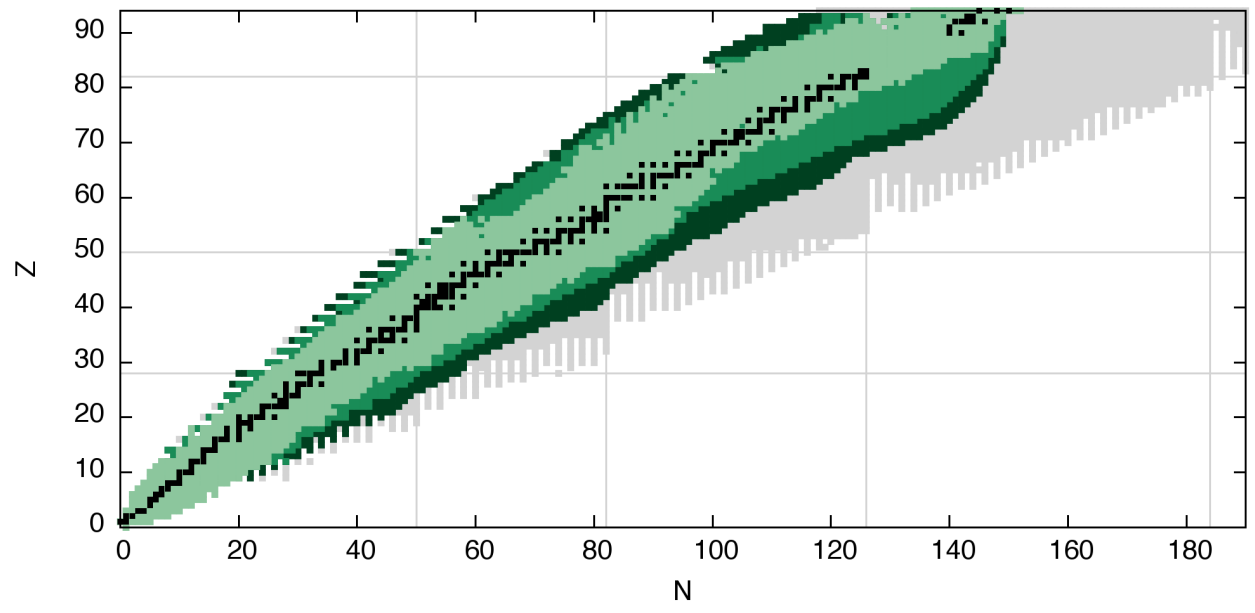

Figure 1. Nuclear chart highlighting the anticipated FRIB reach, showing stable nuclei (black), nuclei with measured masses included in the 2016 Atomic Mass Evaluation [11] (light green squares), nuclei with masses estimated to be measurable at FRIB on Day 1 (green squares), and nuclei with masses estimated to be measurable once FRIB reaches its designed beam intensity (dark green squares). FRIB rates are from [16]. Here we take a mass to be measurable if the stopped beam rate is anticipated to be greater than $10^{-3} \mathrm{pps}$ and the predicted half-life is greater than $10 \mathrm{~ms}$. The grey shaded region indicates where $S_{n}>0$ and $S_{p}>0$ as calculated using FRDM2012 nuclear masses.

The current extent of mass measurements is shown in Fig. 1, where the isotopes with experimental mass values appearing in the 2016 Atomic Mass Evaluation [11] are highlighted in light green. Outside of this region, $r$-process simulations rely on theoretical mass models. Different models tend to agree fairly well where data is available and diverge markedly away from this region (see, e.g, Fig. 6 of [6]). Separation energy predictions among different models can disagree by MeV (see, e.g., Fig. 3 of [12]). The extent of positive values of neutron separation energies for one popular mass model, the Finite Range Droplet Model (FRDM2012) [15], is shown as the light grey shaded region in Fig. 1. All of the nuclei on the neutron-rich side of this region can potentially participate in a neutron star merger $r$-process.

Fig. 1 also highlights the upcoming mass measurement capabilities anticipated for FRIB [16]. Beam requirements depend on the nature of the mass measurement technique. Here we estimate a mass to be measurable if FRIB can achieve a stopped beam intensity of approximately $10^{-3} \mathrm{pps}$, and if the isotope has a estimated or measured half-life of at least $10 \mu \mathrm{s}$. When FRIB begins operations in 2020, the planned Day 1 beam intensity of $10 \mathrm{~kW}$ puts the experimental reach for mass measurements as shown in the green shaded region of Fig. 1. The ultimate design intensity of FRIB at $400 \mathrm{~kW}$ results in a predicted reach given by the dark green shaded region of Fig. 1.

It is clear from Fig. 1 that dedicated experimental campaigns at FRIB can result in an unprecedented number of new mass measurements. This has the potential to radically transform the capability of $r$-process simulations to provide precision estimates of nucleosynthetic abundances and heating rates. To roughly illustrate this, we perform Monte Carlo variations of uncertain nuclear masses in an $r$-process simulation. We choose astrophysical conditions similar to those expected for a heated neutron star merger accretion disk wind, with entropy $s / k \sim 87$, dynamical timescale of $80 \mathrm{~ms}$, and initial electron fraction $Y_{e}=0.26$. These con- 


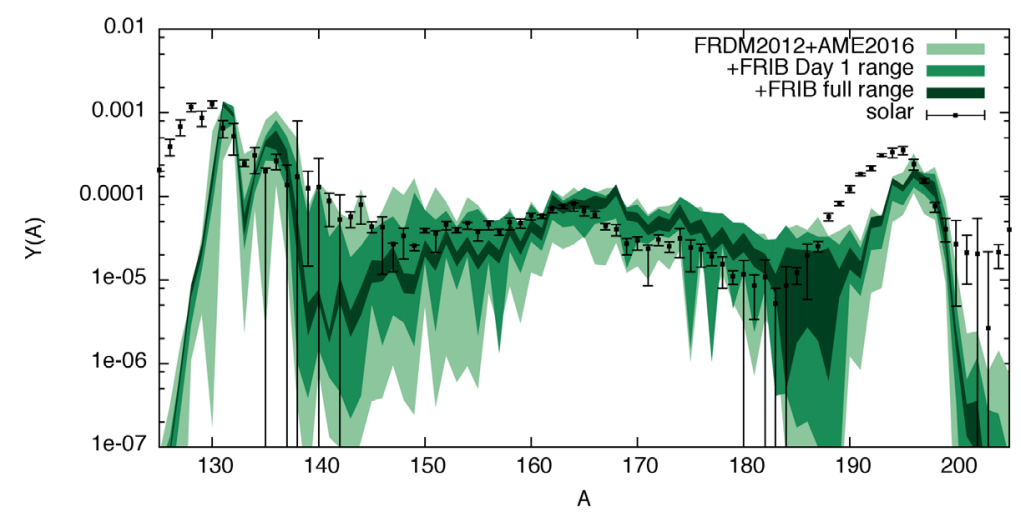

Figure 2. Abundance pattern $1 \sigma$ uncertainty bands obtained from 10,000 sets of uncorrelated, random variations of individual nuclear masses outside the range of: the AME2016 (light green band), the FRIB Day 1 anticipated reach (green band), and the FRIB design reach (dark green band), for hot wind astrophysical conditions $\left(s / k=87, \tau=80 \mathrm{~ms}, Y_{e}=0.26\right)$. Points show scaled solar $r$ residuals [5].

ditions produce a hot, $(n, \gamma)-(\gamma, n)$ equilibrium $r$ process, where the influence of the masses is predominantly through the neutron separation energies [13]. Neutron separation energies set the abundances along each isotopic chain while equilibrium holds and influence the individual neutron capture rates and photodissociation rates that are important once equilibrium fails. The $r$-process calculations proceed as in $[6,8]$. The baseline simulation uses AME2016 masses with FRDM2012 masses where experimental values are not available, NUBASE 2016 [14] $\beta$-decay rates with rates from [17] where experimental values are not available, and NONSMOKER [18] neutron capture rates. Fission is treated as in [6], though the conditions chosen do not result in appreciable fission cycling.

The base simulation is repeated 10,000 times, with each step including random, uncorrelated variations of all masses assumed to be unknown. The random mass variations are pulled from Gaussian distributions of width $0.5 \mathrm{MeV}$. The latter choice is guided by the typical $\sigma_{R M S}$ values of the mass models most commonly used in $r$-process calculations, $\sigma_{R M S} \sim 0.4-1.0$ $\mathrm{MeV}$. For the chosen astrophysical conditions the greatest influence of the mass is through the photodissociation rates, which set the equilibrium abundances and compete with neutron capture and $\beta$-decay in freezeout. Photodissociation rates are calculated by detailed balance and thus have an exponential dependence on separation energy; mass changes of $\sim 0.5 \mathrm{MeV}$ can result in changes to photodissociation rates as large as two orders of magnitude. In contrast, the same mass changes produce changes to neutron capture rates of factors of two to five and $\beta$-decay rates of factors of two to four [13]. Thus to capture the primary influence of masses for these astrophysical conditions, we update the neutron separation energies and photodissociation rates for each step as in [8]. The light green shaded region of Fig. 2 shows the $1-\sigma$ variation in the resulting 10,000 abundance patterns when all of the masses outside of the AME2016 are randomly varied in this way. The width of the shaded band is similar to that found in past studies of uncorrelated mass variations [22] and in recent studies of systematic mass variations [4]. To estimate the improvements expected from dedicated FRIB measurement campaigns, we repeat the full set of 10,000 simulations twice, where we restrict the range of random nuclear mass variations to (1) outside the Day 1 FRIB reach and (2) outside the full FRIB reach. The resulting abundance pattern variations are shown in the green and dark green bands, respectively, of Fig. 2. Note significant improvements to the abundance 


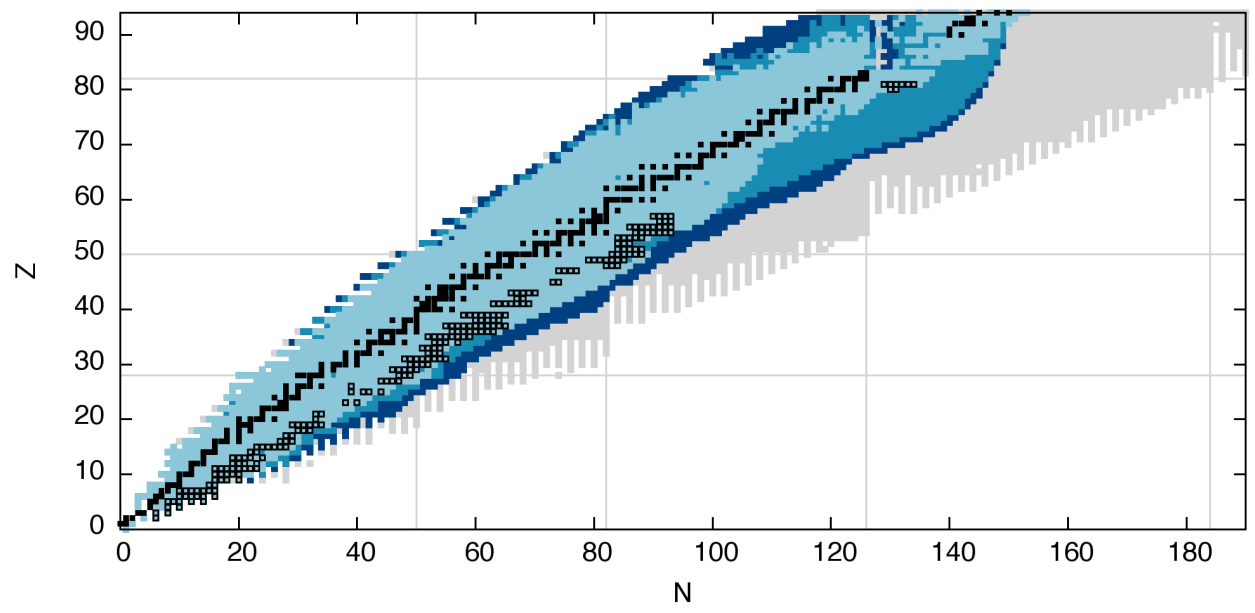

Figure 3. Nuclear chart highlighting the anticipated FRIB reach, showing stable nuclei (black), nuclei with measured half-lives from the NUBASE 2016 compilation (light blue squares), nuclei with measured $P_{n}$ from NUBASE 2016 (open black squares), nuclei with half-lives estimated to be measurable at FRIB on Day 1 (blue squares), and nuclei with half-lives estimated to be measurable once FRIB reaches its designed beam intensity (dark blue squares). FRIB rates are from [16]. Here we take a half-life to be measurable if the stopped beam rate is anticipated to be greater than $10^{-5} \mathrm{pps}$. The grey shaded region is defined as in Fig. 1.

pattern uncertainties can be realized with FRIB at $10 \mathrm{~kW}$ intensity, and the potential improvement with FRIB at full intensity is dramatic. Fine details of the abundance pattern that are currently obscured by uncertainties in nuclear masses can potentially be clearly resolved.

The calculations presented in Fig. 2 consider only the role of nuclear masses in a hot, $(n, \gamma)-(\gamma, n)$ equilibrium $r$ process. For a cold or very neutron rich $r$-process, photodissociation plays a more limited role and thus the full uncertainties in $r$-process abundances due to nuclear masses can only be captured when mass changes are propagated through to the neutron capture rates and $\beta$-decay lifetimes [13]. Monte Carlo studies for these conditions are presented in [12]. Additional uncertainties come from the uncertainty in nuclear structure of neutron-rich nuclei, which shapes the level densities and $\gamma$-strength functions required for neutron capture rate calculations and the $\beta$-strength functions needed for $\beta$-decay lifetimes and $\beta$-delayed neutron emission estimates. FRIB has the potential to dramatically reduce these uncertainties as well.

Lifetimes for $\beta$ decay determine the relative abundances of the different isotopic chains for an $r$-process in $(n, \gamma)-(\gamma, n)$ equilibrium and govern the decay back to stability once equilibrium fails. Thanks in part to recent measurement campaigns at RIKEN [19,20] more experimental values are available for $\beta$-decay lifetimes than any other set of nuclear data. This is illustrated in Fig. 3, where the nuclei with experimental half-lives compiled in NUBASE 2016 are shown in light blue. Outside this region, $r$-process simulations use lifetimes calculated from gross theory or with some application of the quasiparticle random phase approximation (QRPA), e.g., [9, 17, 21]. Interestingly, the variations among different approaches which reach orders of magnitude for long-lived nuclei narrow far from stability to approximately a factor of two [9], as $Q$-values increase and the lifetimes become less sensitive to details of the estimated nuclear structure. Monte Carlo determinations of $r$-process abundance pattern 
uncertainties due to this factor of two uncertainty in $\beta$-decay rates are presented in Fig. 17 in [9].

The reach of FRIB for new lifetime measurements is also shown in Fig. 3, for the Day 1 and design beam intensities. The improvements to $r$-process uncertainties due to extending lifetime measurements to the FRIB full range are not anticipated to be as dramatic as in the case of nuclear masses for the reasons mentioned above; the current half-life data extends further into neutron-rich regions than the mass data and the theoretical model disparities are not as large. Instead the importance shifts to providing new measurements of lifetimes near the $N \sim 126$ closed shell region, reducing the errors on existing measurements, and measuring the probabilities $P_{n}$ that $\beta$ decay is followed by the emission of one or more neutrons. Also of key importance is the determination of nuclear structure information via the $\beta$-decay strength functions [23]. Nuclei close to the drip line will always be out of reach of experiment, and lifetimes alone are insufficient to constrain theory. $P_{n}$ measurements and improvements to lifetimes beyond a factor of two are required to study the fine details of the final abundance pattern, for example, in the rare earth peak [22], see also Fig. 14 of [9].

Precision predictions of $r$-process abundance patterns and careful interpretation of kilonova signals require improvements in our understanding of nuclear physics far from stability. Here we examined the part that the Facility for Rare Isotope Beams is poised to play in realizing these improvements. For nuclear masses, dedicated experimental campaigns for direct measurements alone have the potential to result in dramatic improvements in $r$-process abundance predictions. For $\beta$ decay, the greatest need is to go beyond lifetime measurements to investigate $\beta$-strength functions and $\beta$-delayed neutron emission probabilities [24]. Also important to note is the promise of FRIB to contribute to unknown neutron capture rates through transfer reaction [25] and $\beta$-Oslo [7] measurements. We encourage FRIB to fully embrace its potential role as the next-generation $r$-process machine in planning for Day 1 experiments and beyond.

Acknowledgements This work was supported in part by U.S. Department of Energy under contract No. DE-SC0013039 (RS) and by the National Nuclear Security Administration of the U.S. Department of Energy at Los Alamos National Laboratory under Contract No. DEAC52-06NA25396 (MM).

\section{References}

[1] B.P. Abbott et al. Phys. Rev. Lett. 119, 161101, (2017)

[2] P.S. Cowperthwaite et al., Astrophys. J. Lett. 848, L17 (2017).

[3] D. Kasen, B. Metzger, J. Barnes, E. Quataert, E. Ramirez-Ruiz, Nature 551, 80 (2017)

[4] B. Côté, C.L. Fryer, K. Belczynski, O. Korobkin, M. Chruślińska, N. Vassh, M.R. Mumpower, J. Lippuner, T. M. Sprouse, R. Surman, R. Wollaeger, accepted in Astrophys. J., arXiv:1710.05875 (2018)

[5] M. Arnould, S. Goriely, K. Takahashi, Phys. Rep. 450, 97 (2007)

[6] M.R. Mumpower, R. Surman, G.C. McLaughlin, A. Aprahamian Prog. Part. Nucl. Phys. 86, 86 (2016)

[7] S.N. Liddick et al., Phys. Rev. Lett. 116, 242502 (2016)

[8] R. Surman, M. Mumpower, A. Aprahamian, Acta Phys. Polonica B 47, 673 (2016)

[9] T. Shafer, J. Engel, C. Fröhlich, G.C. McLaughlin, M. Mumpower, R. Surman, Phys. Rev. C 94, 055802 (2016)

[10] J. Barnes, D. Kasen, M.-R. Wu, G. Martínez-Pinedo, Astrophys. J. 829, 110 (2016)

[11] M. Wang, G. Audi, F. G. Kondev, W. J. Huang, S. Naimi, X. Xu, Chinese Phys. C 41 030003 (2017) 
[12] R. Surman, M. Mumpower, G.C. McLaughlin, 14th International Symposium on Nuclei in the Cosmos (NIC2016) 010612 (2017)

[13] M. Mumpower, R. Surman, D.-L. Fang, M. Beard, P. Möller, T. Kawano, A. Aprahamian, Phys. Rev. C 92, 035807 (2015)

[14] G. Audi, F.G. Kondev, M. Wang, W.J. Huang, S. Naimi, Chinese Phys. C 41,030001 (2017)

[15] P. Möller, A.J. Sierk, T. Ichikawa, H. Sagawa, At. Data Nucl. Data Tables 109, 1 (2016)

[16] https://groups.nscl.msu.edu/frib/rates/fribrates106.html

[17] P. Möller, B. Pfeiffer, K.-L. Kratz, Phys. Rev. C 67, 055802 (2003)

[18] T. Rauscher, F.-K. Thielemann, At. Data Nucl. Data Tables 75, 1 (2000)

[19] G. Lorusso et al., Phys. Rev. Lett. 114, 192501 (2015)

[20] J. Wu et al., Phys. Rev. Lett. 118, 072701 (2017)

[21] T. Marketin, L. Huther, G. Martínez-Pinedo, Phys. Rev. C 93, 025805 (2016)

[22] M.R. Mumpower, G.C. McLaughlin, R. Surman, A.W. Steiner, J. Phys. G 44, 034003 (2017)

[23] A. Spyrou et al., Phys. Rev. Lett. 117, 142701 (2016)

[24] R. Grzywacz et al., FRIB Decay Station white paper, in preparation

[25] J.A. Cizewski, A. Ratkiewicz, J.E. Escher, A.Lepailleur, S.D. Pain, G. Potel, EPJ Web of Conferences 165, 01013 (2018) 